\title{
Semitone frequency mapping to improve music representation for nucleus cochlear implants
}

\author{
Sherif Abdellatif Omran ${ }^{1,2^{*}}$, Waikong Lai ${ }^{1}$, Michael Büchler ${ }^{1}$ and Norbert Dillier ${ }^{1}$
}

\begin{abstract}
The frequency-to-channel mapping for Cochlear implant (Cl) signal processors was originally designed to optimize speech perception and generally does not preserve the harmonic structure of music sounds. An algorithm aimed at restoring the harmonic relationship of frequency components based on semitone mapping is presented in this article. Two semitone (Smt) based mappings in different frequency ranges were investigated. The first, Smt-LF, covers a range from 130 to $1502 \mathrm{~Hz}$ which encompasses the fundamental frequency of most musical instruments. The second, Smt-MF, covers a range from 440 to $5040 \mathrm{~Hz}$, allocating frequency bands of sounds close to their characteristic tonotopical sites according to Greenwood's function. Smt-LF, in contrast, transposes the input frequencies onto locations with higher characteristic frequencies. A sequence of 36 synthetic complex tones (C3 to B5), each consisting of a fundamental and 4 harmonic overtones, was processed using the standard (Std), Smt-LF and Smt-MF mappings. The analysis of output signals showed that the harmonic structure between overtones of all complex tones was preserved using Smt mapping. Semitone mapping preserves the harmonic structure and may in turn improve music representation for Nucleus cochlear implants. The proposed semitone mappings incorporate the use of virtual channels to allow frequencies spanning three and a half octaves to be mapped to 43 stimulation channels. A pitch difference limen test was done with normal hearing subjects discriminating pairs of pure tones with different semitone intervals which were processed by a vocoder type simulator of $\mathrm{Cl}$ sound processing. The results showed better performance with wider semitone intervals. However, no significant difference was found between 22 and 43 channels maps.
\end{abstract}

Keywords: Semitone mapping, Melody, Music, Nucleus cochlear implant

\section{Introduction}

Music can be described as a series of complex acoustic sounds composed of tones with fundamentals and overtones that are harmonically related to each other [1]. The majority of musical instruments generate fundamental frequencies below $1 \mathrm{kHz}$ [2]. An important aspect of music is melody [3] which can be defined as a sequence of individual tones that are perceived as a single entity [4]. Preserving the harmonic structure of individual tones is important for preserving the melody perception.

Cochlear Implants (CIs) were originally designed to restore speech perception for patients with profound hearing loss $[5,6]$. The standard ACE (advanced

\footnotetext{
* Correspondence: sherif.omran@gmx.de

'ENT Department, University Hospital Zurich, Frauenklinikstrasse 24, CH-8091 Zurich, Switzerland

Full list of author information is available at the end of the article
}

combination encoder) speech coding strategy used with the Nucleus CI typically encodes signals between 188 and $7980 \mathrm{~Hz}$ onto maximally 22 intracochlear electrodes. The frequency range up to $1 \mathrm{kHz}$ is represented by only up to eight electrodes in the standard (Std) ACE frequency to electrode mapping. This is insufficient to preserve the representation of the harmonic structure of musical tones, because the fundamental frequencies as well as overtones of adjacent musical tones will often be mapped onto the same electrode, especially for frequencies below $500 \mathrm{~Hz}$. It can be hypothesized therefore that this coding strategy will not be optimal for musical melody representation.

One way to improve tonotopic melody representation would be to ensure that the fundamental frequencies of adjacent tones on the musical scale are assigned to separate electrodes. Such an approach involves mapping fundamental frequencies of musical tones to electrodes

\section{SpringerOpen ${ }^{\odot}$}

(C) 2011 Omran et al; licensee Springer. This is an Open Access article distributed under the terms of the Creative Commons Attribution License (http://creativecommons.org/licenses/by/2.0), which permits unrestricted use, distribution, and reproduction in any medium, provided the original work is properly cited. 
based on a semitone scale. The idea was initially investigated in a study by Kasturi and Loizou [7], using the 12 electrode Clarion CII (Advance Bionics) implant with a limited range of semitone frequencies. They concluded that semitone spacing improved melody recognition with CI recipients. Additionally, music could be further enhanced by increasing the frequency representation in CIs. This may be possible by using virtual channels (VCs) formed by stimulating two adjacent electrodes simultaneously with the same current level. Busby and Plant reported that VCs invoked the perception of an intermediate pitch [8]. VCs on an array of 22 electrodes would yield a total number of 43 channels, which would allow covering three and a half octaves with semitone (Smt) mapping with one-semitone intervals between the characteristic frequencies of successive channels. Note that the middle $\mathrm{VC}$ between two adjacent electrodes is the only $\mathrm{VC}$ which can be created in present Nucleus CI devices, because they are equipped with only one current source.

In this study, we extended Kasturi and Loizou's idea and propose a Smt mapping algorithm incorporating VCs. Two different Smt mapping ranges were considered. The first one, Smt-LF, is restricted to the low and mid frequency range [130 to $1502 \mathrm{~Hz}$ ] and the second, Smt-MF, maps frequencies in the mid and high frequency range [440 to $5009 \mathrm{~Hz}$ ]. The ranges of Smt-LF and Smt-MF mappings in relation to a piano keyboard are illustrated in Figure 1. Note that at the lower end of the piano scale, the fundamental frequencies of successive tones differ by as little as $3 \mathrm{~Hz}$ at A0 $\left(f_{0}=27 \mathrm{~Hz}\right)$ and approx. $8 \mathrm{~Hz}$ at $\mathrm{C} 3\left(f_{0}=130 \mathrm{~Hz}\right)$. This difference

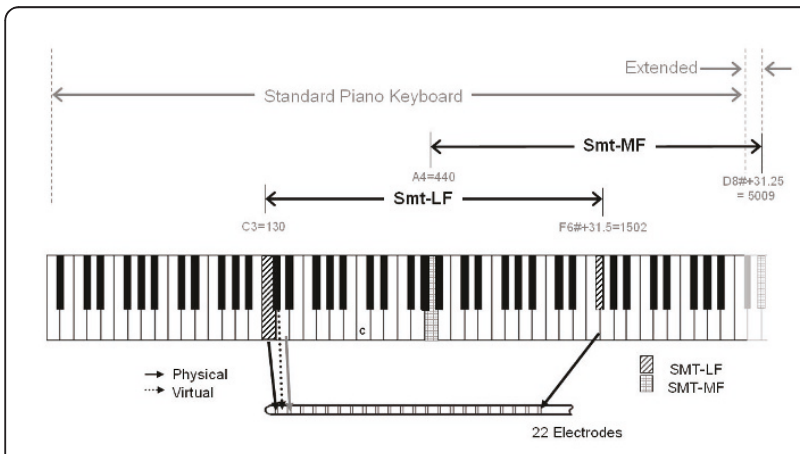

Figure 1 With Smt mapping, consecutive tones on the musical scale (illustrated here using a piano keyboard) are assigned to adjacent channels corresponding to either physical electrodes (solid lines) or virtual channels (dotted lines). An array of 22 physical electrodes yields a total of 43 channels. Smt-LF and Smt-MF respectively map the tones from C3 to F6\#, and from A4 to D8\#, to these channels. Note that the tones C8\# to D8\# (shaded keys) have been added for illustration purposes only and do not exist on the standard piano keyboard. increases as the fundamental frequency of the tone is also increased.

The frequency range of the Smt-LF mapping covers low and mid frequencies. These frequencies are common to most musical instruments [2]. The Smt-LF mapping has a band-pass filter that filters out the fundamental frequencies and the lower partials lying within the first and second piano octaves (less than 130 $\mathrm{Hz}$ ) as well partials in the sixth piano octave and above (greater than $1502 \mathrm{~Hz}$ ). The range of the Smt-MF mapping covers part of the mid and high frequencies that are common between music and comprehensible speech bands used in telephone lines [9]. The Smt-MF mapping band-pass filters out frequencies lower than $440 \mathrm{~Hz}$ (A4) and higher than $5009 \mathrm{~Hz}$. Thus fundamental frequencies and partials in most of the fourth piano octave (261 to $493 \mathrm{~Hz}$ ) and below will not be represented. Smt-MF mapping also allocates frequency bands of audible sounds to electrodes with similar characteristic frequencies according to Greenwood's formula [10,11] assuming an average cochlear length of $33 \mathrm{~mm}$ and electrode insertion depth of $22 \mathrm{~mm}$.

This article is organized as follows: 'Theoretical basis of semitone mapping' section describes the theoretical basis of semitone mapping and explains why Smt mapping was chosen. 'Processing and implementation' section presents a brief description of the processing technique. 'Semitone mapping frequency ranges' section describes in detail the two Smt-LF and Smt-MF ranges in concern. 'Frequency time matrix' section describes how the resolution at low frequencies was improved. 'Channel time matrix' section describes how the frequency bands were then mapped to their corresponding channels. 'Nucleus Matlab Toolbox' section is a description of how an acoustic model could be implemented to resynthesize channel activities into an acoustic sound that will be used in psychoacoustic tests with normal hearing $(\mathrm{NH})$ listeners. 'Analysis' section shows an analysis for the resynthesized sounds. 'Pilot test' section describes a pilot pitch ranking test using acoustic simulations of $\mathrm{CI}$ sounds with $\mathrm{NH}$ subjects to investigate the perceptual difference between 43 and 22 channels using the Std ACE mapping. The hypothesis for this test being that 43 Channel mode would increase the frequency representation, and as a result enhance synthetic tone discrimination and improve pitch ranking with smaller semitone intervals than with 22 channels using the Std ACE mapping. 'Procedure' and 'Results' sections describe the experimental procedures and results. This is followed by a discussion and a conclusion section.

\section{Theoretical basis of semitone mapping}

Smt mapping assigns fundamental frequencies of successive semitones on the musical scale to individual 
channels. Note that the harmonic overtones, which are integer multiples of the fundamental frequency, of each musical tone will also be mapped to the center frequency of separate channels with Smt mapping. Therefore, different musical tones will correspond to different sets of channels.

The relationship between the fundamental frequencies $f_{n}$ and $f_{r}$ of two musical tones $k$ semitones apart is described by Equation 1a below.

$$
\begin{aligned}
& f_{n}=f_{r} \cdot 2^{k / 12} \\
& \Rightarrow \log _{10}\left(\frac{f_{n}}{f_{r}}\right)=0.025 k
\end{aligned}
$$

where $f_{r}$ is the fundamental frequency of the lower tone. Equation 1b represents the ratio of characteristic frequencies of channels in Smt mapping. Substituting $k$ $=1$ gives frequency ratios for one-semitone steps.

The characteristic frequencies of Smt mapping for 43 channels each 1 semitone apart $(k=1)$ for the two SmtLF and Smt-MF ranges (squares and filled circles, respectively) are plotted in Figure 2. Note that higher channel numbers correspond to lower frequencies, to be consistent with the numbering used for Nucleus CIs. The characteristic frequencies of the Std mapping with 43 channels (i.e., including VCs) are also shown in Figure 2 (open circles).

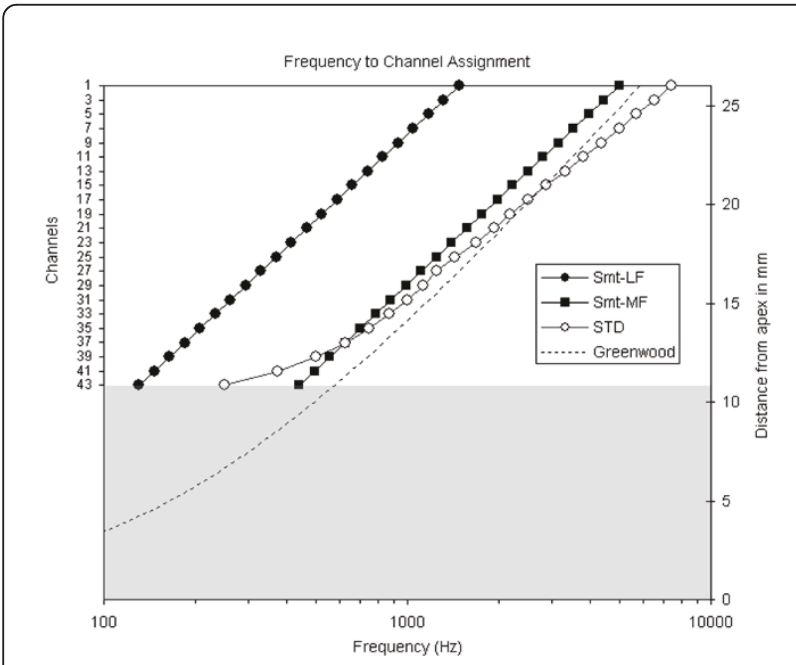

Figure 2 The frequency to channel assignments for the Smt-LF (filled circles), Smt-MF (filled squares) and Std (open circles) mappings are shown here together with the Greenwood function (dashed line, secondary $y$-axis). The figure assumes a cochlea length (unrolled) of $33 \mathrm{~mm}$, and illustrates an insertion depth of $22 \mathrm{~mm}$ for a Nucleus straight array with 22 equally spaced electrodes. The channel location within the cochlea can be derived from the two $y$-axes.
The two Smt mapping functions yield straight lines with a slope corresponding to the value of 0.025 as given in Equation 1b. This value is required to map consecutive semitones to consecutive individual channels. Shallower slopes would result in more than one semitone being mapped to the same channel, distorting the original harmonic structure of the overtones. This would be the case for the Std mapping function, particularly with the first eight channels in the lower frequency range. This distortion decreases at higher frequencies as the slope approaches a value corresponding to 0.025 .

Since the inner ear resolves frequencies mainly based on a logarithmic function, harmonic overtones with the Smt mapping will be regularly spaced along the basilar membrane as described by the following equations.

Equation 2 below describes the characteristic frequencies at distance $x \mathrm{~mm}$ from the cochlea's apex according to Greenwood's empirically derived function which was verified against data that correspond to a range of $x$ from 1 to $26 \mathrm{~mm}$ [12].

$$
\begin{aligned}
& f(x)=165.4\left(10^{0.06 \cdot x}-1\right) \\
& \Rightarrow x(f)=\frac{1}{0.06} \log \left(\frac{f}{165.4}+1\right)
\end{aligned}
$$

The distance (in $\mathrm{mm}$ ) between two locations with different characteristic frequencies $f_{1}$ and $f_{2}$ is given by Equation 4

$$
\Delta x=x_{2}-x_{1}=\frac{1}{0.06} \log \left(\frac{f_{2}+165.4}{f_{1}+165.4}\right)
$$

Substituting $f_{2}$ and $f_{1}$ by $f_{n}$ and $f_{r}$, respectively, from Equation 1 yields:

$$
\Delta x=\frac{1}{0.06} \log \left(\frac{2^{k / 12}+165.4 / f_{r}}{1+165.4 / f_{r}}\right)
$$

Equation 5 shows that the spacing along the basilar membrane between two successive semitones (substitute $k=1$ and $f_{r}$ with the fundamental frequency of the lower tone) will vary depending on the frequency range, and is smaller at low frequencies, asymptotically approaching $0.4 \mathrm{~mm}$ with higher frequencies. For $\mathrm{C} 3\left(f_{0}\right.$ $=130.8 \mathrm{~Hz}$ ), the spacing would be about $0.19 \mathrm{~mm}$, whereas at $\mathrm{C} 8\left(f_{0}=4186 \mathrm{~Hz}\right)$ about $0.4 \mathrm{~mm}$. The electrode spacing between successive electrodes in the Nucleus 24 implant straight array has a center to center distance of $0.75 \mathrm{~mm}$ [13]. For VCs, assuming that the center of stimulation is halfway between the two physical electrodes, the channel spacing would be about 0.38 $\mathrm{mm}$. This corresponds roughly to the tonotopical spacing for the tones involved in the Smt-MF mapping. 


\section{Processing and implementation}

The block diagram in Figure 3 shows the Std ACE processing algorithm. An acoustic signal undergoes fast Fourier transform (FFT), from which the power spectral density (PSD) is calculated. The frequency range of the PSD is divided into different bands. The $n$ bands with the highest energies (maximas) are then selected for presentation, where $n$ is a parameter that can be defined for each CI recipient's map. The resulting frequency time matrix (FTM) is then processed as follows: The energy within each selected band is used to determine the corresponding stimulation level according to a loudness growth function (LGF). Using a mapping function, the respective bands are then assigned to channels, which can be physical electrodes or VCs, to produce the channel time matrix (CTM).

\section{Semitone mapping frequency ranges}

The fundamental frequencies of the musical tones from the piano keyboard vary between $27.5 \mathrm{~Hz}$ (A0) and 4186 $\mathrm{Hz}$ (C8) [2]. Two ranges were investigated for the Smt mapping:

\section{Smt-LF [130 to $1502 \mathrm{~Hz}$ ] (C3 to F6\#)}

The minimum required frequency resolution for the Smt-LF mapping is approx. $8 \mathrm{~Hz}$ at C3 $\left(f_{0}=130 \mathrm{~Hz}\right)$. Analyzing a signal that has a sampling rate of $16 \mathrm{kHz}$ with 2048 FFT points provides a $7.8-\mathrm{Hz}$ resolution between successive frequency bins. The lowest acoustic frequency of $130 \mathrm{~Hz}$ for Smt-LF will be mapped to the most apical electrode location, which would correspond to a characteristic tonotopical frequency of approximately $571 \mathrm{~Hz}$ estimated according to Greenwood's equation [10], assuming an average cochlear length of $33 \mathrm{~mm}$ and an electrode array insertion depth of 22 $\mathrm{mm}$. This will cause sounds to be perceived higher in pitch. However, as the frequency shift is expected to be the same for all partials with Smt mapping, this would be equivalent to a transposition.

Smt-MF [440 to $5009 \mathrm{~Hz}$ ] (A4 to D8\#)

A small frequency bandwidth is enough to maintain speech comprehension as in telephone transmission, where the bandwidth used is [300 to $3000 \mathrm{~Hz}$ ] [9]. The Smt-MF mapping covers part of the bandwidth that is common between speech and music [440 to $5009 \mathrm{~Hz}$ ]. Note that transposing the Smt-MF range three semitones higher to cover a range from $523 \mathrm{~Hz}$ (C5) to 5919 $\mathrm{Hz}$ (F8\#) would minimize the difference between characteristic and tonotopical frequencies of electrodes according to Greenwood [10] (see Figure 2) assuming an average cochlear length of $33 \mathrm{~mm}$ and an insertion depth of $22 \mathrm{~mm}$. However, it is impossible to precisely match the tonotopical characteristic frequencies for any given individual through Greenwood's function as the latter is empirical in nature and is also supposed to only represent the average $\mathrm{NH}$ listener. Also cochlear length and electrode insertion depth vary among patients. Thus, some discrepancy is always to be expected.

\section{Frequency time matrix}

Frequency components at different time frames are analyzed using FFT and are organized into a FTM. A typical CI processor like the Nucleus Freedom uses a sampling rate $f_{s}$ of $16 \mathrm{kHz}$ to produce the FTM with a 128 points FFT $[14,15]$, giving a frequency resolution $\Delta f$ of $125 \mathrm{~Hz}$ [16-18]. However, Smt-LF mapping needs a higher resolution ( $\Delta f$ of approx. $8 \mathrm{~Hz}$ ) at low frequencies (approx. $130 \mathrm{~Hz}$ ). Increasing the number of points $N=$ $f_{S} / \Delta f$ increases the frequency resolution but at the same time will produce smearing in the time domain due to the larger processing window. In order to increase the frequency resolution at low frequencies and retain some of the time resolution at higher frequencies, frequency subband decomposition [19-21] is used to generate the FTM.

\section{Frequency resolution and subband decomposition}

First, the input signal is sampled at $16 \mathrm{kHz}$. Then the sampled signal is processed in two frequency subbands (see Figure 4) to yield two different frequency resolutions.

Figure 4 shows how the input signal flows into two parallel pathways: one for the low frequencies and the other for the high frequencies. The low frequency pathway uses $512(N)$ samples which are split into overlapping time frames and analyzed. The amount of overlap depends on the stimulation rate such that at the end of each stimulation period, as much new data (sampled at $16 \mathrm{kHz}$ ) as needed is added to the data buffer. For instance, with a stimulation rate of $500 \mathrm{~Hz}, 32$ new samples are added every stimulation period to the data buffer of length 512 samples, resulting in an overlap of 480 samples. The signal is first filtered using a Kaiser LPF with a cutoff at $4 \mathrm{kHz}$, and then decimated by a factor of two $(d=2)$ which increases the frequency resolution

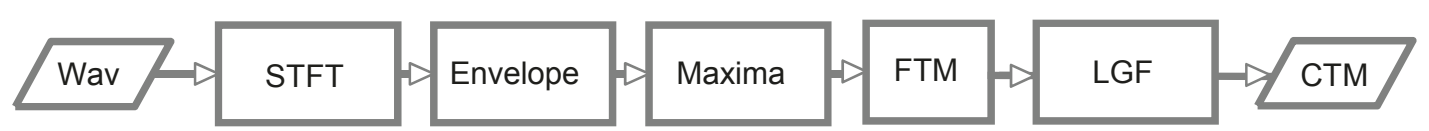

Figure 3 Processing block diagram illustrating the standard ACE coding strategy for the Nucleus cochlear implant. 


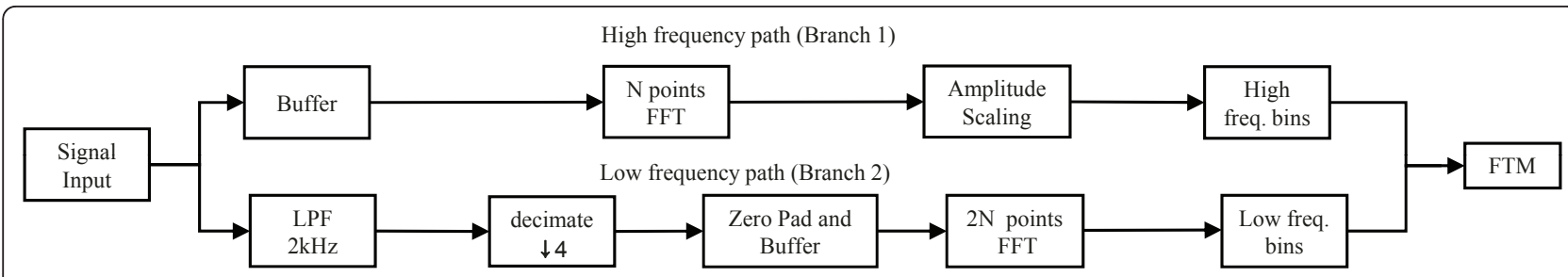

\section{Zero Pad}

Length $=2^{\mathrm{n}}$

Figure 4 Implementation of frequency subband decomposition for Smt-LF, producing a frequency resolution of $7.8 \mathrm{~Hz}$ at frequencies below $1054 \mathrm{~Hz}$ (LF path), and a frequency resolution of $31.25 \mathrm{~Hz}$ at higher frequencies (HF path). The number of overlapping points between frames is calculated as follows: Overlap $=512-f_{s} /$ (stimulation rate). Note that the analysis frame rate is the same as the stimulation rate.

by the same factor while keeping the buffer length 512 points. Each time frame with 512 points is Hanning filtered and zero padded before undergoing a $2048(\mathrm{~m})$ point FFT. Notice that after zero padding each bin represents a frequency band of $3.9 \mathrm{~Hz}\left(f_{\mathrm{s}} / m=8 \mathrm{k} /\right.$ 2048). Every two successive bins are then summed to preserve the power and decrease the overall minimum detectable frequency difference $\left(\Delta f=f_{s} /(d \cdot m / 2)\right)$ in the low frequency branch within successive bands to $7.8 \mathrm{~Hz}$.

The high frequency pathway uses the same number of points $(N=512)$ used in the low frequency pathway, producing a frequency resolution of 31.25 . The signal is split into overlapping time frames in the same manner as in the low frequency pathway. Each time frame is processed with a Hanning filter of the same number of points, zero padded and undergoes a 2048 point FFT.

The output bins from both pathways are combined to form the FTM which has a bin resolution of 7.8. The boundary between the low and the high frequency pathways was set to $1054 \mathrm{~Hz}$ (between C6 and C6\#) where the difference in frequency between successive semitones starts to exceed the HF resolution. This ensures that any successive semitones will at least lie on successive electrodes. The lower 134 bins are from the low frequency pathway, while the higher bins are from the high frequency pathway.

An example of a FTM produced using frequency subband decomposition for a signal with four sinusoidal components with $900,936,1200$, and $1295 \mathrm{~Hz}$ is shown in Figure 5. The difference in frequency resolution can be clearly seen in the narrower bands at lower frequencies and wider bands at higher frequencies.

The above frequency subband decomposition only applies to the Smt-LF mapping. For Smt-MF mapping, the minimum frequency resolution required is approx. $26.6 \mathrm{~Hz}$ at A4 $\left(f_{0}=440 \mathrm{~Hz}\right)$. Using $N=512$ provides a minimum resolution of $31.25 \mathrm{~Hz}$ which is slightly larger than the required resolution for the lowest semitone frequencies. Note that in the present implementation of the Smt-MF, the first two tones (A4 and A4\#) will not be adequately resolved and therefore fall within a single FFT bin which will in turn be mapped to two adjacent channels because the difference between them is less than the LF resolution (7.8 $\mathrm{Hz}$ ). To preserve the starting frequency and approach

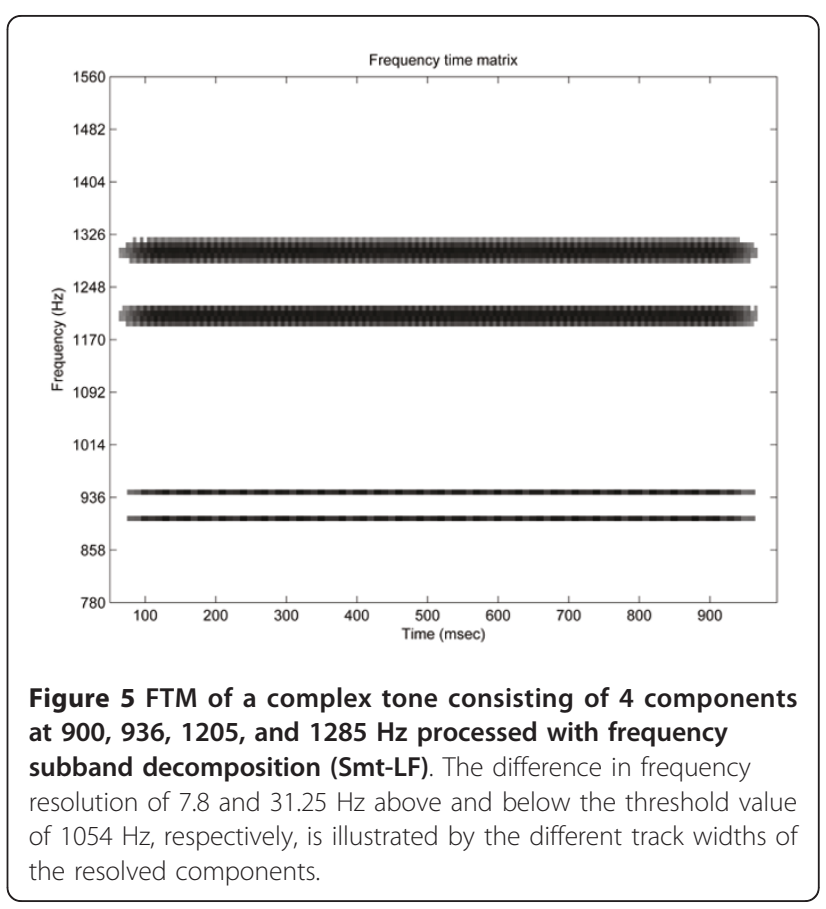


CFs to Greenwood frequencies without having an empty channel, while having frequencies of (A4 and A4\#) semitones being in the same bin, it is suggested to activate the first two electrodes. The starting frequency could have been made slightly lower, but a drawback would produce a bigger difference between CFs of electrodes and Greenwood frequencies. The remaining tones are adequately resolved. Subband decomposition is not used with Smt-MF mapping. The processing block diagram for Smt-MF is similar to the one described for the high frequency pathway in Figure 4 , with $N=512$ without zero padding and without the frequency scaling block. Note that the frequency resolution of Smt-MF is $31.25 \mathrm{~Hz}$, compared to $7.8 \mathrm{~Hz}$ (for frequencies below $1054 \mathrm{~Hz}$ ) and $31.25 \mathrm{~Hz}$ (for frequencies above $1054 \mathrm{~Hz}$ ) for Smt-LF.

\section{Channel time matrix}

Depending on the frequency range of interest (e.g. SmtLF [130 to $1502 \mathrm{~Hz}$ ] and Smt-MF [440 to $5009 \mathrm{~Hz}$ ]), different bins in the FTM are combined into frequency channels to produce a CTM. A mapping matrix (M) is introduced to define which FFT bins should be mapped to which corresponding channels. The mapping matrix attempts to map the center frequencies of the channels and FFT bins as close as possible to the fundamental frequencies of each corresponding semitone.

$$
C T M=M \cdot F T M
$$

Figure $6 \mathrm{a}, \mathrm{b}$ illustrates the mapping matrices for both the Smt-MF and the Smt-LF mapping, respectively, for 43 channels. The Smt-LF mapping covers frequency band [130 to $1502 \mathrm{~Hz}$ ] which corresponds to bin numbers [17 to 200], with a frequency resolution of $7.8 \mathrm{~Hz}$. The Smt-MF mapping covers frequency band [440 to $5009 \mathrm{~Hz}$ ] which corresponds to bin numbers [15 to 169] where the frequency resolution is $31.25 \mathrm{~Hz}$. Smt-MF mapping does not incorporate subbands and accordingly bin 15 (corresponding to $440 \mathrm{~Hz}$ ) is mapped to channels 1 and 2.
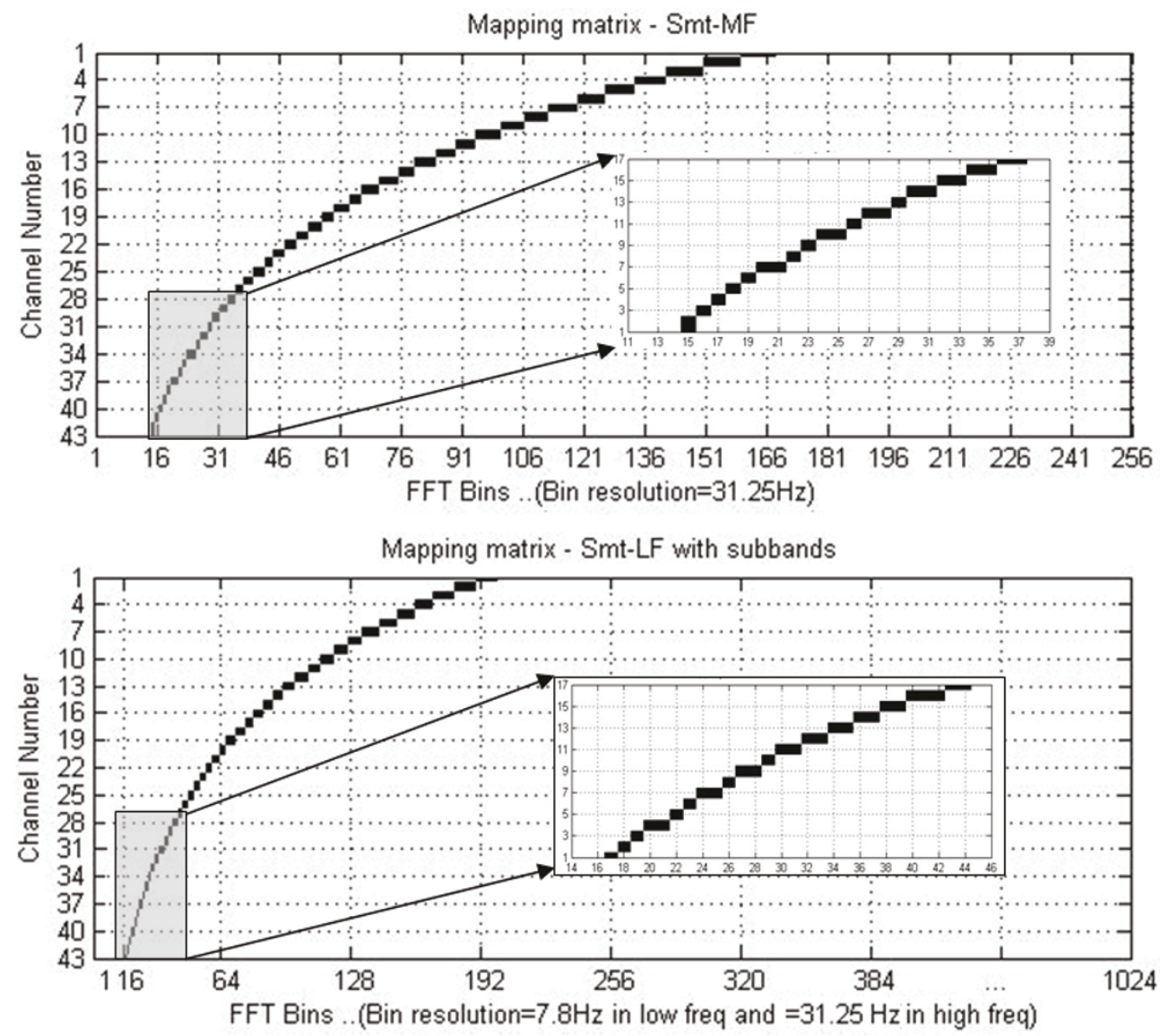

Figure 6 Mapping matrices for Smt-LF (upper) and Smt-MF (lower) with 43 channels 


\section{Nucleus Matlab Toolbox}

The Smt mapping follows the ACE strategy in selecting the highest $n$ channels. It was implemented in Matlab and incorporated into the Nucleus Matlab Toolbox (NMT) framework [15]. The acoustic model (AMO) was based on noise band vocoders [22]. The activity in each channel is simulated as a white noise convolved with an exponentially decaying filter, where its center frequency is the characteristic frequency of the channel. Channel interactions arising from the spread of the electric field from its center at the stimulation site can be set by a "width of stimulation" parameter. The resulting stimulation of the auditory nerve, causing also the perception of adjacent pitches, is simulated with the AMO. In the Smt-LF mapping, the AMO simulated the frequency transposition.

\section{Analysis}

Following the definition of [4] for melody, a better representation of individual musical tones is expected to ameliorate melody recognition, or in other words, melody is poorly resolved if individual musical tones are poorly represented. Musical tones are characterized by their harmonic structure. To compare the harmonic structure representation for the three different mappings (Std, Smt-MF, and Smt-LF), a sound sequence consisting of 36 consecutive synthetic musical tones was constructed. Each tone consisted of five partials with successive $20 \%$ decrease in amplitude and lasting for $150 \mathrm{~ms}$. The fundamental frequency of each tone increased from $130 \mathrm{~Hz}$ (C3) to $987 \mathrm{~Hz}$ (B5) with 1-semitone interval.

Figure 7 shows the harmonic structure being preserved with both Smt-MF and Smt-LF mappings, where the spacing of the partials remains uniform across tones. With the Std mapping at low frequencies, partials are not resolved. With Smt-MF, frequency components below $440 \mathrm{~Hz}$ are filtered out (as indicated by arrows in Figure 7), while with Smt-LF, the high frequency partials greater than $1.6 \mathrm{kHz}$ are filtered out.

\section{Pilot test}

A pitch difference limen test was conducted using a pair of single harmonic pure tones with 1, 3, and 6 semitone intervals. Tones were preprocessed with a $\mathrm{CI}$ acoustic model [23] that uses a noise band vocoder in the resynthesis algorithm. A CI acoustic resynthesis model was used to simulate the sound CI patients may perceive and to present these to $\mathrm{NH}$ subjects. The model assumes that there is no change in the effective spread of excitation width between 43 and 22 channels. Pure tones were used that corresponded to fundamental frequencies of musical tones. All tones were modified to have the same temporal envelope, and a duration of 0.5 s. The starting and ending of all tones were faded with $30 \mathrm{~ms}$ attack and release times simultaneously. The reference note $(D)$ was used for all tone groups $(1,3$, and 6 semitone intervals).

\section{Procedure}

All tones were processed using 22 and 43 channels with the acoustic model using a stimulation width of $1 \mathrm{~mm}$ since $[22,24]$ found that a width of stimulation of around $1 \mathrm{~mm}$ produced electrode discrimination similar to that of average Nucleus CI24 recipients. Sound samples were then normalized to have equal loudness. $\mathrm{NH}$ subjects were seated in front of a loudspeaker at a distance of $1.5 \mathrm{~m}$ and sounds were presented at a level of $70 \mathrm{dBA}$. MACarena [25] software was used to randomly select and play a pair of two tones from three octave groups: octave 3,4 , and 5 . The tone pairs from each octave group were D-D\#, D-F, and D-G\#, with 1,3 , and 6 semitone intervals, respectively. The randomization was to minimize learning effects of tone sequences. For each group the same number of repetitions was presented. The tone pairs were presented sequentially with a pause in between of $0.5 \mathrm{~s}$. Levels were roved by \pm 6 $\mathrm{dB}$ to avoid loudness cues from being used. Eight $\mathrm{NH}$ subjects aged between 27 and 55 years took part in this experiment.

\section{Results}

The results of the pitch difference limen measurements are summarized in Figure 8 and show that all subjects, as expected, demonstrated improved pitch discrimination performance with increasing semitone intervals. An ANOVA test showed that there was no statistical difference between 22 and 43 channels at a significance level of $95 \%$.

\section{Discussion}

CIs have limitations arising from the physical size of the electrode array and the insertion depth which contribute to CI recipients' difficulty to recognize melodic sounds. Furthermore, the available 22 electrodes of the Nucleus $\mathrm{CI}$ are expected to be too few to provide a good representation of melodies. Drennen et al. report that with monopolar stimulation, recipients perceive pitch based on a degraded spatial representation, suggesting that only 8 to 9 distinguishable channels of the available 22 may exist [26]. To increase the number of channels, VCs [8] are considered here.

Preserving the harmonic structure of musical notes is expected to ameliorate melody representation of musical sounds with CIs. This article investigates the preservation of the harmonic structure by employing semitone mapping with two new ranges together with the use of VCs. The frequency distribution of a semitone mapping 

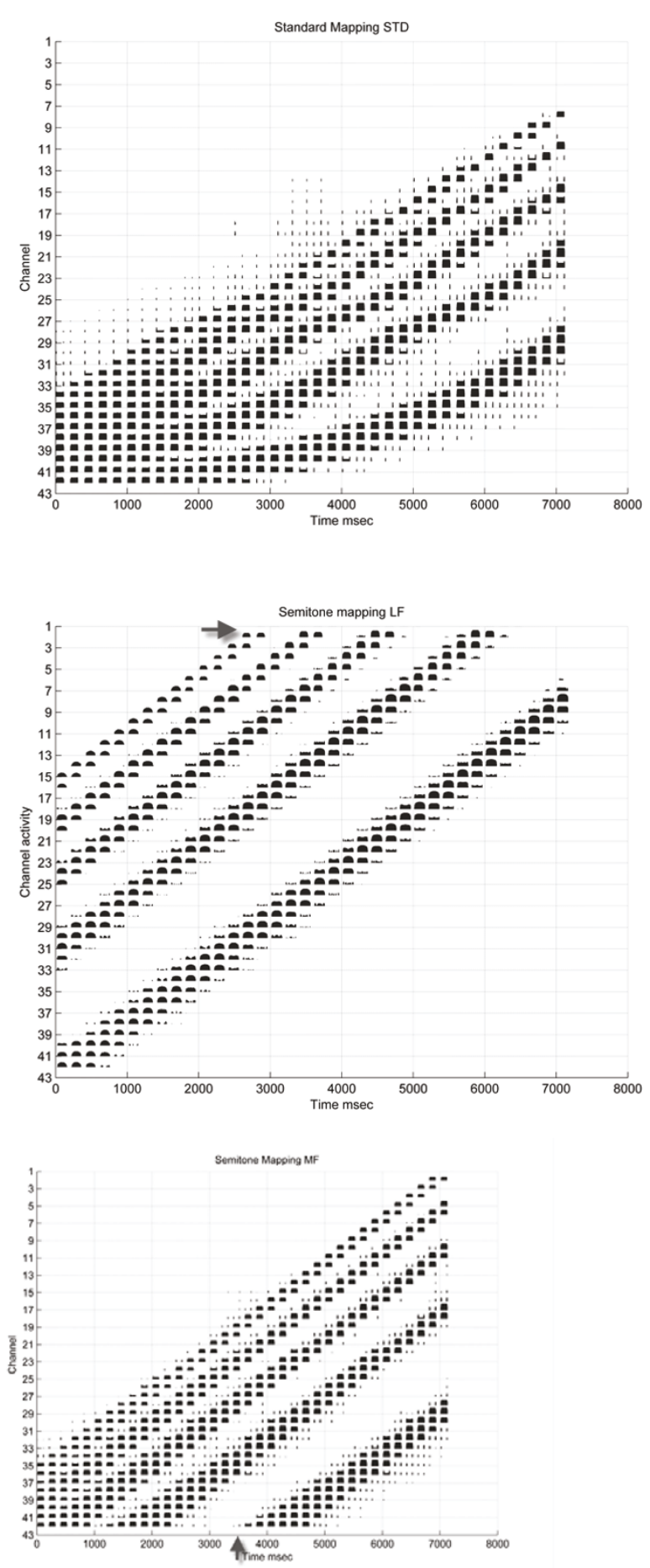

Figure 7 CTM outputs for the Std (a), Smt-LF (b) and Smt-MF (c) mapping, for a sequence of 36 synthetic musical tones (C3 to B5) each consisting of the first 5 partials and lasting $\mathbf{1 5 0} \mathbf{m s}$. The $x$-axis also represents the tones ordered along the musical scale. It can be seen that with Smt-LF and Smt-MF, the relationship between partials (as illustrated by the logarithmically distributed channels along the $y$-axis) remains the same for all input tones. With the Std mapping, the relationship between partials varies depending on the pitch of the input tone, and at lower frequencies, the partials cannot even be resolved. 


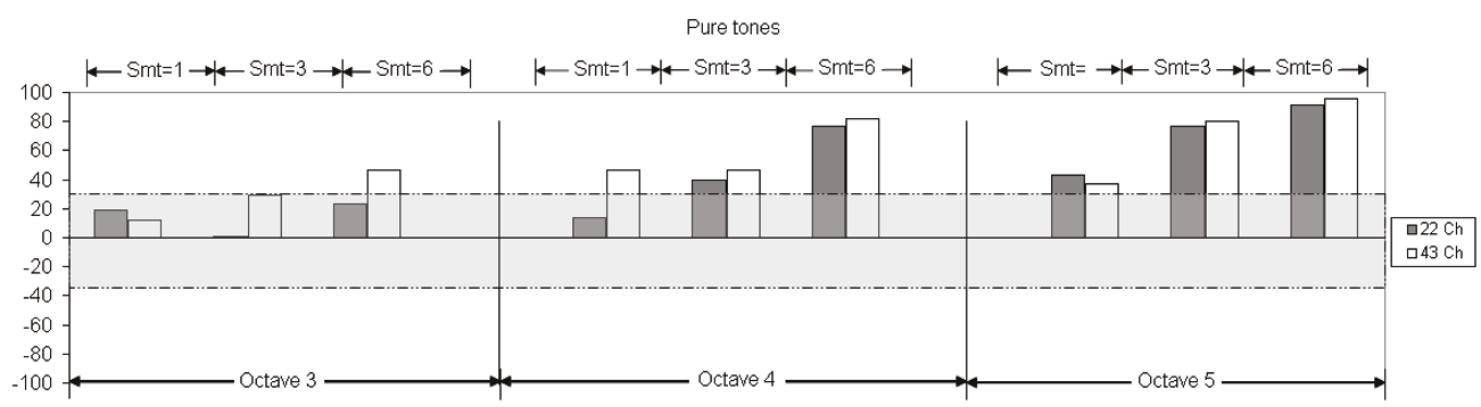

Figure 8 Results of 22 (grey) and 43 channels (white) for 1, 3, and 6 semitones intervals in octaves 3, 4, and 5 using pure tones. The region between the dotted lines represents chance level.

is determined by the number of semitones that are to be mapped over the number of available channels. When incorporating VCs, the characteristic frequency of each channel is one semitone apart from the previous one. If VCs were not used (i.e., only the original 22 physical channels (electrodes) are used instead), the characteristic frequency of each channel would be two semitones apart, in order to cover the same input frequency range.

The Smt-LF mapping [130 to $1502 \mathrm{~Hz}$ ] needs a resolution of approx. $8 \mathrm{~Hz}$ at the low frequency end $(130 \mathrm{~Hz})$. Although the resolution can be increased by increasing the number of points in the FFT analysis, this would also cause the signal to be smeared in the time domain. A compromise is achieved by using frequency subbands, in which different frequency bands can have different resolutions. With Smt-LF, frequencies below $1054 \mathrm{~Hz}$ are analyzed with a resolution of approx. $8 \mathrm{~Hz}$ while higher frequencies are analyzed with a $31.25-\mathrm{Hz}$ resolution. Note that the frequency allocation for each channel will not be exact for each semitone due to the discrete nature of the FFT bin outputs. There is a limitation with the Smt-LF mapping in that frequencies higher than $1502 \mathrm{~Hz}$ as well as frequencies lower than $130 \mathrm{~Hz}$ are filtered out. This might lead to filtering out some fundamental frequency that could result in pitch reversals of tones or degradations in the perceived sound quality. In the Smt-LF mapping, frequencies are transposed into higher ranges compared to their tonotopic characteristic frequencies, making tones sound higher in pitch. It is expected that CI patients with Smt-LF mapping may distinguish low frequencies better but music may not sound pleasant.

In Smt-MF mapping, the characteristic frequencies of channels approach their tonotopical frequencies according to the Greenwood function. Consequently, Smt-MF mapping may sound more natural than Smt-LF mapping. Smt-MF does not need the use of subbands analysis when a FFT size of 512 points is used as a single resolution of $31.25 \mathrm{~Hz}$ is sufficient (except for the lowest two tones). There is however a limitation with the Smt-
MF map at low frequencies, where components below $440 \mathrm{~Hz}$ are filtered out. This filtering may remove the fundamental frequency of some tones which could then result in pitch reversals compared to tones that have all frequency components intact.

An exact matching of the electrode location to the tonotopical characteristic frequency location is not possible [27]. Thus the phenomenon of the missing fundamental is not expected to occur with CI patients. Equation 4 yields a range of 0.19 to $0.38 \mathrm{~mm}$ for the spacing between successive semitones for the Smt-LF mapping. For the Smt-MF mapping, the range is from 0.3 to $0.4 \mathrm{~mm}$. Assuming that VCs are located exactly midway between two adjacent physical electrodes, the channel spacing for the Nucleus straight electrode array is about $0.38 \mathrm{~mm}$. This is the same order of magnitude as the spacing required for the Smt-MF mapping. Although the match is not exact, the semitone mapping minimizes the spatial distortion of the harmonic structure representation on the basilar membrane.

Semitone mapping leads to the stimulation of different groups of electrodes for each tone on the musical scale. Each tone has a harmonic structure of overtones which will in turn be regularly spaced along the basilar membrane. The analysis results showed that Smt mapping preserved the spatial ratio of partials of the processed tones. This may lead to a better preservation of the harmonic structure when presented to a CI recipient. The mapping approach outlined in this article has been tested with $\mathrm{NH}$ listeners as well as $\mathrm{CI}$ recipients and the outcomes are presented in an accompanying article [28].

The results from the pilot study comparing 22 versus 43 channels suggest that tones with higher semitone intervals are easier to identify than smaller semitone intervals in octaves 3,4 , and 5 . However, the results are not statistically different. Kwon and van den Honert [29] have reported that intermediate pitches can also be achieved using non-simultaneous stimulation of adjacent electrodes, provided that they are sequentially stimulated 
in a time less than the nerve refractory period. Thus, an explicit use of VCs may not be necessary to achieve intermediate pitch sensations. Based on these observations, it was decided that subsequent testing of the standard and semitone mappings as reported in the companion article [28] would use 22 channels.

The algorithm illustrated in this paper follows the regular signal processing methods as in the Std mapping algorithm, but the FFT processing and the subbands blocks may be optimized using the $\mathrm{S}$ transform [30] for limited memory speech processors. It may be also worthwhile to investigate the effect of other transforms such as constant Q transform [31] where the frequency domain is decomposed into logarithmic constant bands with a constant number of semitones per octave. Derivatives of this transform may be considered as well such as modified constant $\mathrm{Q}$ fast filter bank transform [32].

Smt mapping may be one possible step on a path to improve music representation with CI devices by preserving the harmonic structure of overtones to enhance melody representation, but other aspects of music such as timbre should also be considered as well. Timbre depends partially on the representation of temporal fine structure [33]. Including fine structure analysis in new strategies may improve music representation $[26,34,35]$ but this is beyond the scope of the present Smt mapping development. In the companion paper [28], several psychoacoustic tests with $\mathrm{NH}$ and CI patients were conducted with the proposed strategies. They showed that semitone mapping enhanced melody contour identification and significantly improved the recognition of certain instruments. The analysis of the output signals presented in this article confirms that semitone mapping preserves well the harmonic structure of synthetic harmonic musical tones [36].

\section{Conclusion}

Semitone mapping is able to better preserve the representation of the harmonic structure of overtones compared to the standard mapping used in the speech processor. This is expected to improve melody recognition with cochlear implant recipients. However, limited frequency range could result in pitch reversals for tones whose high and low frequency partials are filtered out. The pitch difference limen test results showed that the performance of normal hearing subjects with pure tones processed using an acoustic model of the CI output was not statistically significantly different for 43 channels compared to 22 channels, using the standard ACE frequency to channel mapping.

\section{List of abbreviations}

ACE: advanced combination encoder; AMO: acoustic model; Cl: Cochlear implant; CTM: channel time matrix; FTM: frequency time matrix; LGF: loudness growth function; NH: normal hearing; NMT: Nucleus Matlab Toolbox; Smt: semitone; Std: standard; VCs: virtual channels.

\section{Acknowledgements}

The work was supported by Swiss National Science Foundation Grant Number 320000-110043.

\section{Author details}

'ENT Department, University Hospital Zurich, Frauenklinikstrasse 24, CH-8091 Zurich, Switzerland ${ }^{2}$ Institute of Neuroinformatics, University of Zurich, Winterthurerstrasse 190, CH-8057 Zurich, Switzerland

\section{Competing interests}

The authors declare that they have no competing interests.

Received: 23 December 2010 Accepted: 21 June 2011

Published: 21 June 2011

\section{References}

1. F Salzer, Structural Hearing: Tonal Coherence in Music. vol. 1. (Dover Publications, New York, 1982)

2. J Pierce, The Science of Musical Sound (Scientific American Books, New York, 1983)

3. S Sadie, G Grove, The New Grove Dictionary of Music and Musicians. (Grove London, 1995)

4. E Terhardt, Akustische Kommunikation. (Springer-Verlag, Berlin, 1998)

5. G Clark, Cochlear Implants: Fundamentals and Applications. Modern Acoustics and Signal Processing vol. XXXVIII. Springer-Verlag, Heidelberg, 2003)

6. K Gfeller, S Witt, J Stordahl, M Mehr, G Woodworth, 2000, The effect of training on melody recognition and appraisal by adult cochlear implant recipients. J Acad Rehabil Audiol. 23, 115-138 (2000)

7. K Kasturi, $\mathrm{P}$ Loizou, Effect of filter spacing on melody recognition: acoustic and electric hearing. J Acoust Soc Am. 122(2), EL29-34 (2007)

8. PA Busby, KL Plant, Dual electrode stimulation using the nucleus CI24RE cochlear implant: electrode impedance and pitch ranking studies. Ear Hear. 26(5), 504-511 (2005)

9. S Möller, Quality of Telephone-Based Spoken Dialogue Systems. (Springer, US, 2005)

10. D Greenwood, A cochlear frequency-position function for several species29 years later. J Acoust Soc Am. 87(6), 2592-2605 (1990)

11. D Greenwood, Critical bandwidth and consonance in relation to cochlear frequency-position coordinates. Hear Res. 54(2), 164-208 (1991)

12. D Greenwood, Critical bandwidth and the frequency coordinates of the basilar membrane. J Acoust Soc Am. 33(10), 12 (1961)

13. LT Cohen, Practical model description of peripheral neural excitation in cochlear implant recipients: 1. Growth of loudness and ECAP amplitude with current. Hear Res. 247(2), 87-99 (2009)

14. B Swanson, E Van Baelen, M Janssens, M Goorevich, T Nygard, K Van Herck, Cochlear implant signal processing ICs. IEEE 2007 Custom Intergrated Circuits Conference (CICC). San Jose, CA, (2007)

15. B Swanson, Pitch Perception with Cochlear Implants. in Faculty of Medicine, Dentistry \& Health Sciences, Otolaryngology Eye and Ear Hospital. (University of Melbourne, Melbourne, 2008), p. 299

16. MS Fourakis, JW Hawks, LK Holden, MW Skinner, TA Holden, Effect of frequency boundary assignment on speech recognition with the Nucleus 24 ACE speech coding strategy. J Am Acad Audiol. 18(8), 700-717 (2007)

17. J Carroll, FG Zeng, Fundamental frequency discrimination and speech perception in noise in cochlear implant simulations. Hear Res. 231(1-2), 42-53 (2007)

18. MS Fourakis, JW Hawks, LK Holden, MW Skinner, TA Holden, Effect of frequency boundary assignment on vowel recognition with the Nucleus 24 ACE speech coding strategy. J Am Acad Audiol. 15(4), 281-299 (2004)

19. RE Crochiere, SA Webber, JL Flanagan, Digital coding of speech in subbands. Bell Syst Tech J. 55(8), 1069-1085 (1976)

20. P Vary, U Heute, W Weiss, in Digitale Sprachsignalverarbeitung. vol. XIII. (B.G. Teubner, Stuttgart, 1998), p. 591

21. S Wyrsch, Adaptive Subband Signal Processing for Hearing Instruments. (ETH Zurich, Zurich, 2000)

22. J Laneau, M Moonen, J Wouters, Factors affecting the use of noise-band vocoders as acoustic models for pitch perception in cochlear implants. J Acoust Soc Am. 119(1), 491-506 (2006) 
23. J Laneau, J Wouters, M Moonen, Improved music perception with explicit pitch coding in cochlear implants. Audiol Neurotol. 11, 38-52 (2006)

24. J Laneau, J Wouters, Multi-channel place pitch sensitivity in cochlear implant recipients. J Assoc Res Otolaryngol. 5, 285-294 (2004)

25. W Lai, N Dillier, MACarena: a flexible computer-based speech testing environment. 7th International Cochlear Implant Conference 2002. Manchester, (2002)

26. WR Drennan, JT Rubinstein, Music perception in cochlear implant users and its relationship with psychophysical capabilities. J Rehabil Res Dev. 45(5), 779-789 (2008)

27. M Dorman, T Spahr, R Gifford, L Loiselle, S McKarns, T Holden, M Skinner, C Finley, An electric frequency-to-place map for a cochlear implant patient with hearing in the nonimplanted ear. J Assoc Res Otolaryngol. 8(2), 234-240 (2007)

28. SA Omran, WK Lai, N Dillier, Pitch ranking Melody contour and instrument recognition tests using two semitone frequency maps for Nucleus Cochlear Implants. EURASIP J Audio Speech Music Process. 2010(2010) [PP 1-16]

29. BJ Kwon, Cvd Honert, Dual-electrode pitch discrimination with sequential interleaved stimulation by cochlear implant users. J Acoust Soc Am. 120(1), EL1-EL6 (2006)

30. RG Stockwell, A basis for efficient representation of the S-transform. in Digital Signal Processing. (2007). vol. 17. Issue 1, Academic Press Inc. Orlando, FL, USA, 2007)(1), , pp. 371-393

31. J Brown, Calculation of a constant $Q$ spectral transform. J Acoust Soc Am. 89(1), 425-434 (1991)

32. FCCB Diniz, I Kothe, SL Netto, LP Biscainho, High-selectivity filter banks for spectral analysis of music signals. EURASIP J Adv Signal Process. 2007(94704), 12 (2007)

33. S Handel, Timbre perception and auditory object formation. Hearing M BC (Academic Press, San Diego (CA), 1995), pp. 425-461

34. K Nie, G Stickney, FG Zeng, Encoding frequency modulation to improve cochlear implant performance in noise. IEEE Trans Biomed Eng. 52(1), 64-73 (2005)

35. F Chen, Y-T Zhang, A novel temporal fine structure-based speech synthesis model for cochlear implant. J Sigpro. 88, 2693-2699 (2008)

36. S Omran, Detecting diagonal activity to quantify harmonic structure preservation with cochlear implant mappings. Int J Robot Autom. 1(5), 100-112 http://www.cscjournals.org/csc/manuscript/Journals/JRA/volume1/ Issue5/JRA-21.pdf (2011)

doi:10.1186/1687-4722-2011-2

Cite this article as: Omran et al:: Semitone frequency mapping to improve music representation for nucleus cochlear implants. EURASIP Journal on Audio, Speech, and Music Processing 2011 2011:2.

\section{Submit your manuscript to a SpringerOpen ${ }^{\circ}$ journal and benefit from:}

- Convenient online submission

- Rigorous peer review

- Immediate publication on acceptance

- Open access: articles freely available online

- High visibility within the field

- Retaining the copyright to your article

Submit your next manuscript at $\gg$ springeropen.com 